\title{
Carpal Tunnel Injections: A Novel Approach Based on Wrist Width
}

\author{
Travis J. Menge ${ }^{1} \quad$ Elizabeth B. Rinker ${ }^{2} \quad$ Kang-Hsien Fan ${ }^{3} \quad$ John J. Block ${ }^{4}$ Donald H. Lee ${ }^{5}$
}

${ }^{1}$ The Steadman Clinic, Vail, Colorado, United States

${ }^{2}$ Louisiana State University Health Sciences Center, New Orleans, Louisiana, United States

3 VICC Cancer Biostatistics Center, Nashville, Tennessee, United States

${ }^{4}$ Department of Radiology and Radiological Sciences, Vanderbilt University Medical Center, Medical Center North, Tennessee, United States

${ }^{5}$ Vanderbilt University Medical Center, Vanderbilt Orthopaedic Institute, Nashville, Tennessee, United States

J Hand Microsurg 2016;8:21-26.

\begin{abstract}
Address for correspondence Donald H. Lee, MD, Vanderbilt University Medical Center, Vanderbilt Orthopaedic Institute, 1215 21st Avenue South, Ste. 3200, Nashville, TN 37232, United States

(e-mail: Donald.H.Lee@Vanderbilt.edu).
\end{abstract}

\begin{abstract}
Keywords

- anatomic location

- carpal tunnel

- safety

- steroid injection

Introduction Carpal tunnel steroid injections (CTIs) have the potential risk of damaging underlying critical structures, including the median nerve (MN), radial artery (RA), and ulnar neurovascular bundle (UB). The purpose of this study was to analyze the safety of a volar radial (VR) and volar ulnar (VU) CTI, using standardized anatomical "safe zones."

Materials and Methods This study was performed on 87 cadaveric arms using a percentage of the total wrist width as a guide for placement of a VR ( 30 and $33 \%$ of total wrist width) and $\mathrm{VU}$ (60 and $66 \%$ of total wrist width) injection.

Results Our results demonstrate a wide range of anatomic variations in the location of these critical neurovascular structures near the carpal canal, indicating that using superficial landmarks alone for CTIs may result in an increased risk of iatrogenic injury to these critical structures.

Discussion We propose a technique using a percentage of total wrist width as a guide for CTIs. Both VR ( $30 \%$ of wrist width) and VU ( $60 \%$ of wrist width) CTIs offer relatively safe and reliable CTI locations to the carpal canal.

Level of Evidence Not applicable/cadaveric study.
\end{abstract}

\section{Introduction}

Injection of corticosteroids into the carpal canal is a welldocumented therapeutic and diagnostic procedure in the nonoperative management of mild-to-moderate carpal tunnel syndrome. ${ }^{1-10}$ Although the procedure is commonly performed and has been shown to be relatively safe, complications may still arise. Improper needle placement can result in injury to the median nerve (MN), ulnar neurovascular bundle (UB), or radial artery (RA), leading to exacerbation of symptoms, permanent neurologic deficit, or vascular injury.

received

July 20, 2015

accepted after revision

March 9, 2016

A variety of recommendations for injection techniques have been made to minimize injury, including injection medial (ulnar) to the palmaris longus (PL) tendon, ${ }^{9,11,12}$ in line with the fourth digit, ${ }^{5,13,14}$ between the PL and flexor carpi radialis (FCR) tendons, ${ }^{15,16}$ between the PL and flexor carpi ulnaris (FCU) tendons, ${ }^{13}$ and through the FCR tendon. ${ }^{14}$ While injections have been shown to be successful in many cases, success is not uniform. One possible explanation for variability in clinical outcomes is inconsistent placement of the injection and improper dispersion of fluid within the carpal canal as a result of utilizing superficial landmarks

(c) 2016 Society of Indian Hand \& Microsurgeons
DOI http://dx.doi.org/ 10.1055/s-0036-1581192. ISSN 0974-3227. 
alone. A better understanding of the anatomical relationship of the underlying structures based on total wrist width could result in more consistent delivery of fluid, while minimizing potential complications.

This study was designed to analyze the safety of a volar radial (VR) and volar ulnar (VU) injection as a function of total wrist width. Although prior studies have utilized the aforementioned landmarks to determine injection location, there is often high variability in these landmarks with respect to the underlying structures. A standardized anatomical technique based on wrist width could further reduce risk to surrounding structures and be easily applied in the clinical setting with no associated increase in cost to the patient or utilization of resources to the provider.

\section{Materials and Methods}

A total of 87 cadaveric upper extremities with no evidence of previous injury or disease to the distal forearm or wrist were used to simulate carpal tunnel injections. Thirty-six wrists were initially dissected to determine the locations of the neurovascular structures and tendons at the level of the distal wrist flexion crease. Total wrist width, defined as the distance from the skin overlying the radial styloid extending to the skin overlying the ulnar styloid, was recorded for each specimen. The overlying skin and portions of underlying forearm fascia were then removed at this level, exposing the volar wrist and forearm structures (-Fig. 1). Dissection of the underlying soft tissues continued until all structures were fully exposed. Starting at the radial aspect of the wrist, the distances from the radial styloid to the center of the RA, FCR tendon, PL tendon, MN, UB, and FCU tendon were measured. Specimen laterality (right vs. left wrists) and gender were

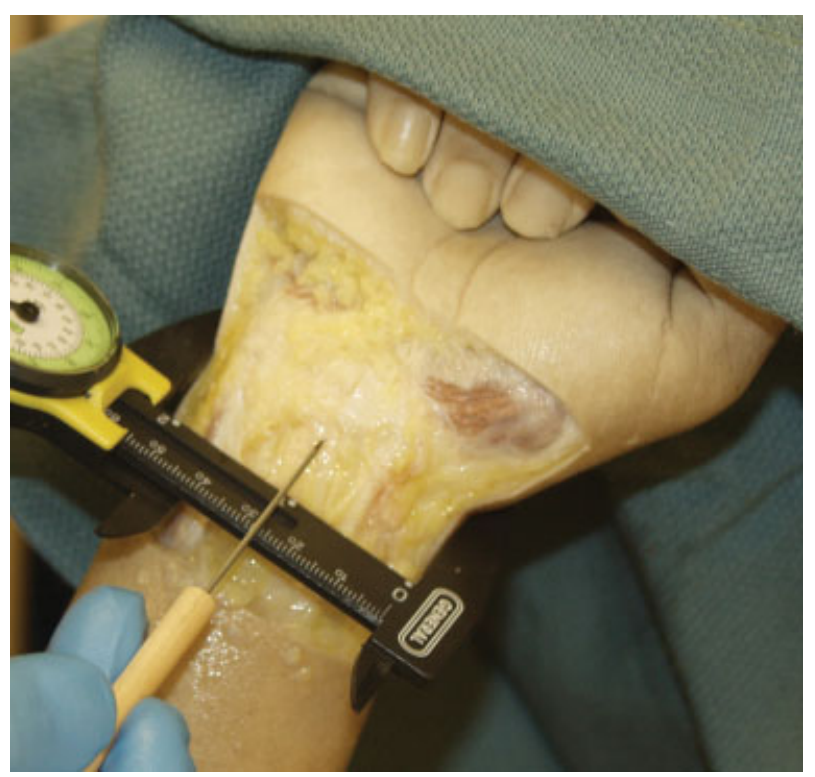

Fig. 1 A view of the volar right wrist following removal of a portion of the skin. A caliper is used to measure the total wrist width (in $\mathrm{mm}$ ) and the distance of the tendons and neurovascular structures from the radial aspect of the wrist. The probe shows the location of and how measurement of the median nerve was taken. recorded. All measurements were taken using a caliper (General Tool Company, Cincinnati, Ohio, United States) and measured in millimeters ( $\mathrm{mm}$; accuracy, $0.01 \mathrm{~mm}$ ).

After documenting the locations of the anatomical structures located at the level of the carpal canal, 32 additional wrists were used to simulate injections and determine the location of the needles in relation to the neurovascular structures. Two 18-gauge hypodermic needles were inserted perpendicular to the wrist at or just proximal to the distal wrist flexion crease, simulating both a VR- and VU-sided carpal tunnel injection into each wrist (-Fig. 2). The first needle was placed into the radial aspect of the wrist at a location reproducing a VR wrist injection, while the second needle was placed at a location reproducing a VU injection. It is important to note that the locations of these needles were based on the previous dissection measurements as a function of total wrist width to attempt to minimize damage to the underlying neurovascular structures.

To reduce inadvertent penetration of these structures, the VR injections were placed at a measured location between the RA and MN, which was calculated as 30 to $33 \%$ of total wrist width. The VU injections, on the other hand, were placed at a measured location between the MN and UB, which again was calculated from our prior findings and found to be 60 to $66 \%$ of total wrist width. All measurements were made from the radial styloid, using the same calipers and previously described techniques. Wrist dissections were performed leaving the needles in situ, thus allowing the locations of the RA, MN, and UB to be recorded relative to needle position. Any penetration of these structures was noted.

To determine the efficacy of these injection sites, a final 19 fresh-frozen cadavers were divided into two groups: VR

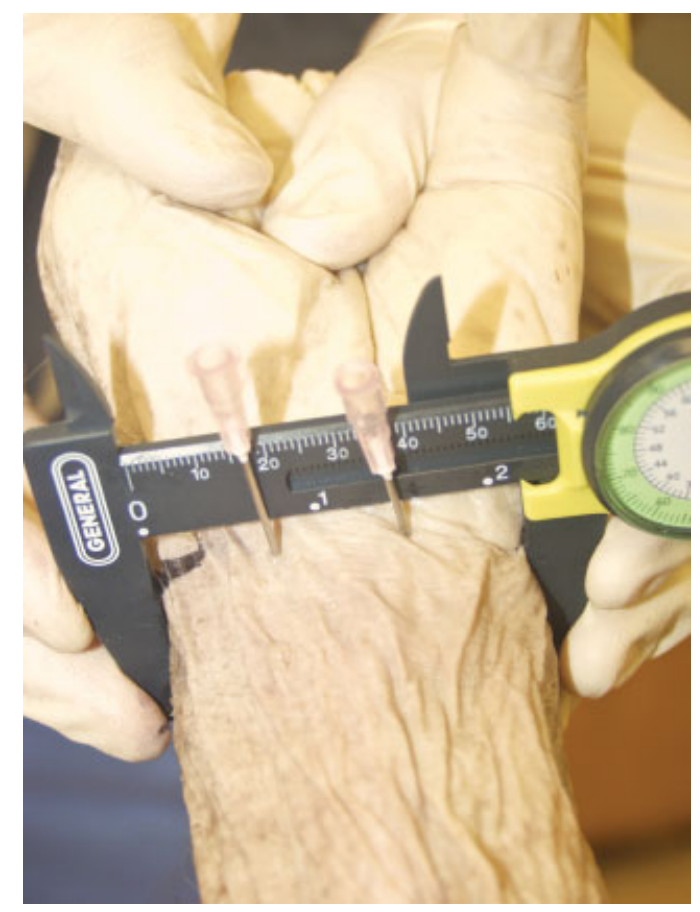

Fig. 2 Figure showing placement of two 18-gauge needles, one at 33\% and the other at $66 \%$ percent of the wrist width as measured from the radial aspect of the wrist. 
versus VU injection groups. Carpal tunnel injections were delivered via a 1.5 -inch, 22 -gauge needle into the canal by one of two techniques by a single injector (D. H. L.). Each injection contained $1 \mathrm{~mL}$ of bacteriostatic normal saline and $1 \mathrm{~mL}$ of Omnipaque 180 radio-opaque dye (GE Healthcare, Princeton, New Jersey, United States). The VR technique (10 wrists) was performed using $30 \%$ of the total wrist width from the radial styloid. The VU technique (nine wrists) was performed using $60 \%$ of the total wrist width. Both injections were performed at the level of or slightly proximal to the wrist flexion crease and directed toward the fourth metacarpal head (radial-sided injection) or index metacarpal head (ulnar-sided injection). Two milliliters of fluid were injected, and the ease of injection was noted. If there was difficulty or resistance while injecting fluid, the needle was partially withdrawn, redirected slightly radially or ulnarly, reintroduced, and fluid injected when free, unobstructed flow was noted.

Following injection, mini C-arm fluoroscopy (OEC Miniview 6800, GE Healthcare-Americas, Salt Lake City, Utah, United States) was used to obtain three images (anteroposterior, lateral, and carpal canal view) of each wrist. These fluoroscopic images were later reviewed by a musculoskeletal radiologist, two attending hand surgeons, and a hand surgery fellow to determine if the injection had been successfully placed within the carpal tunnel. All image readers were blinded as to which injection technique was employed for each set of images.

\section{Statistical Analysis}

Specimen characteristics and location of the RA, MN, and UB are reported as range, mean, and standard deviation (SD) of total wrist width, as appropriate. A Pearson-Clopper statistical method was used to calculate 95\% confidence intervals (CIs) for penetration of these structures by either the radial or ulnar needles. A paired $t$-test was performed to compare right versus left, and a two-sample $t$-test was applied to compare male versus female specimens.

\section{Results}

Data from the initial 36 dissections demonstrate that there was a great deal of anatomic variation among cadaver specimens. The distance of the RA relative to the radial aspect of the wrist ranged from 8 to $28 \%$ of total wrist width (mean: 17\%; SD: 4\%; 95\% CI: $15.4-18.2 \%$ ), MN 31 to 59\% (mean: $46 \%$; SD: 7\%; 95\% CI: 43.8-48.2\%), and UB 63 to 86\% (mean: 72\%; SD: 6\%; 95\% CI: 69.6-73.6\%), respectively. Male specimens demonstrated a statistically significant larger total wrist width compared with female specimens (male: $63.8 \mathrm{~mm}$; female: $58.7 \mathrm{~mm} ; p=0.0004$ ). However, analysis of the locations of the RA, MN, and UB between male and female specimens as a function of total wrist width showed no statistical significance (RA: $p=0.91 ; \mathrm{MN}: p=0.86$; UB: $p=0.24)$. Right-sided specimens were slightly larger than those on the left, but this difference was also not statistically significant (mean: $0.22 \mathrm{~mm} ; p=0.783$ ).

The MN averaged $8.12 \mathrm{~mm}$ in width at the level of the carpal tunnel (range: 6-14; SD: 1.61; 95\% CI: 7.59-8.65). The PL was ulnar to the $\mathrm{MN}$ in 19 subjects $(N=53 \%$; range: $1-31 \mathrm{~mm}$; mean distance: $4.21 \mathrm{~mm}$; SD: $6.71 \mathrm{~mm}$; 95\% Cl: 1.19-7.23 mm), radial in 9 subjects $(N=25 \%$; range: $1-8 \mathrm{~mm}$; mean distance: $5.0 \mathrm{~mm}$; SD: $2.06 \mathrm{~mm}$; 95\% CI: 3.65-6.35 mm), directly overlying the MN in 4 subjects $(N=11 \%)$, and not present in 4 subjects ( $N=11 \%$ ). The FCR was located radial to the MN in all patients and the distance ranged from 2 to $17 \mathrm{~mm}$ (mean: $9.36 \mathrm{~mm}$; SD: $3.63 \mathrm{~mm}$; 95\% CI: 8.18-10.55 mm). There was also a wide range of variation in the locations of the superficial landmarks relative to total wrist width: FCR 19 to 46\%; PL 31 to 95\%; and FCU 71 to $93 \%$.

From these initial results, it was determined radial-sided injections would be placed at $30 \%$, while ulnar-sided injections would be placed at $60 \%$, as both were found to be "safe zones" in our previous dissections. We additionally evaluated one more radial (33\%) and ulnar (66\%) location to determine the effects of moving the injections closer to the MN and UB. Using a radial-sided approach at 30 or $33 \%$, there were no penetrations of either the RA or MN. On the other hand, an ulnar approach at 60\% demonstrated two penetrations of the MN and six penetrations of the UB at 66\%. The ulnar artery was penetrated in four specimens and ulnar nerve in one specimen, and the needle was located between the ulnar nerve and artery in one specimen.

In the final 19 specimens, all injections (19/19) were successfully placed in the carpal canal. A total of 9 were injected from the ulnar side, while 10 were injected from a radial approach. Accurate placement of all injections was confirmed in three-plane fluoroscopy. Each ulnar-sided injection resulted in unobstructed flow with little resistance and showed wide dye dispersion on imaging (-Fig. 3A-C). In four of the radial-sided injections through or near the FCR tendon, there was initially some resistance to the injection requiring redirection of the needle until unobstructed flow was obtained. Fluoroscopic imaging showed dye primarily deposited on the radial side of the canal, and in 8 of the 10 specimens (80\%), a detectable, often substantial amount of dye was found tracking down the flexor pollicis longus (FPL) tendon sheath into the thumb (-Fig. 4A-D).

\section{Discussion}

Carpal tunnel injection is a valuable procedure for diagnostic and therapeutic use in the treatment of carpal tunnel syndrome and is commonly utilized by a wide range of health care providers. Our cadaveric analysis of 87 specimens comparing safety between a VR- and VU-sided injections as a function of total wrist width demonstrates that a VR approach results in a slightly lower incidence of iatrogenic injury to the underlying neurovascular structures compared with a VU approach. There was a wide range of anatomic variation in the relationship between the RA, MN, UB, and surrounding soft tissue structures among specimens, indicating that the use of superficial landmarks alone may result in an increased risk of iatrogenic injury and variability in clinical outcomes. Our standardized anatomical approach based on total wrist width demonstrated significantly less variability than prior techniques. 


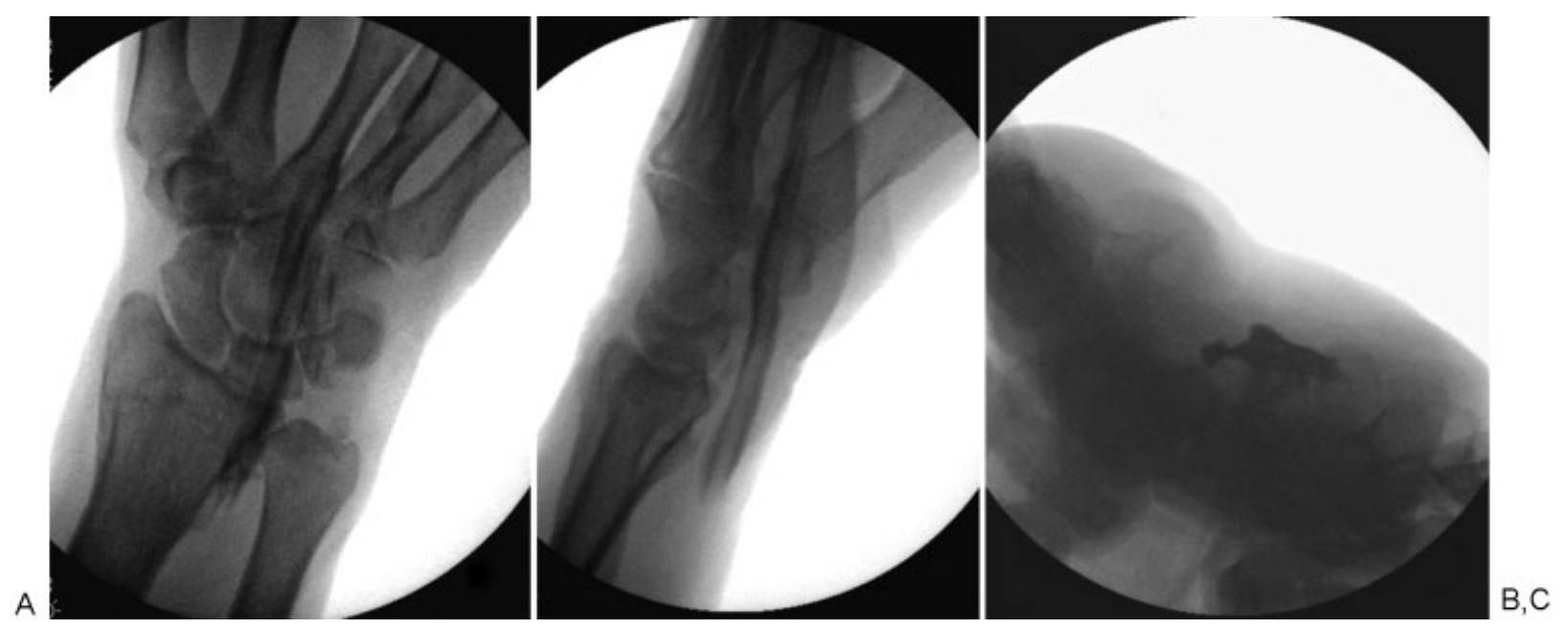

Fig. 3 Fluoroscopic (A) anteroposterior, (B) lateral, and (C) carpal tunnel images after an ulnar-sided injection of the carpal canal showing radioopaque dye located within the carpal canal.

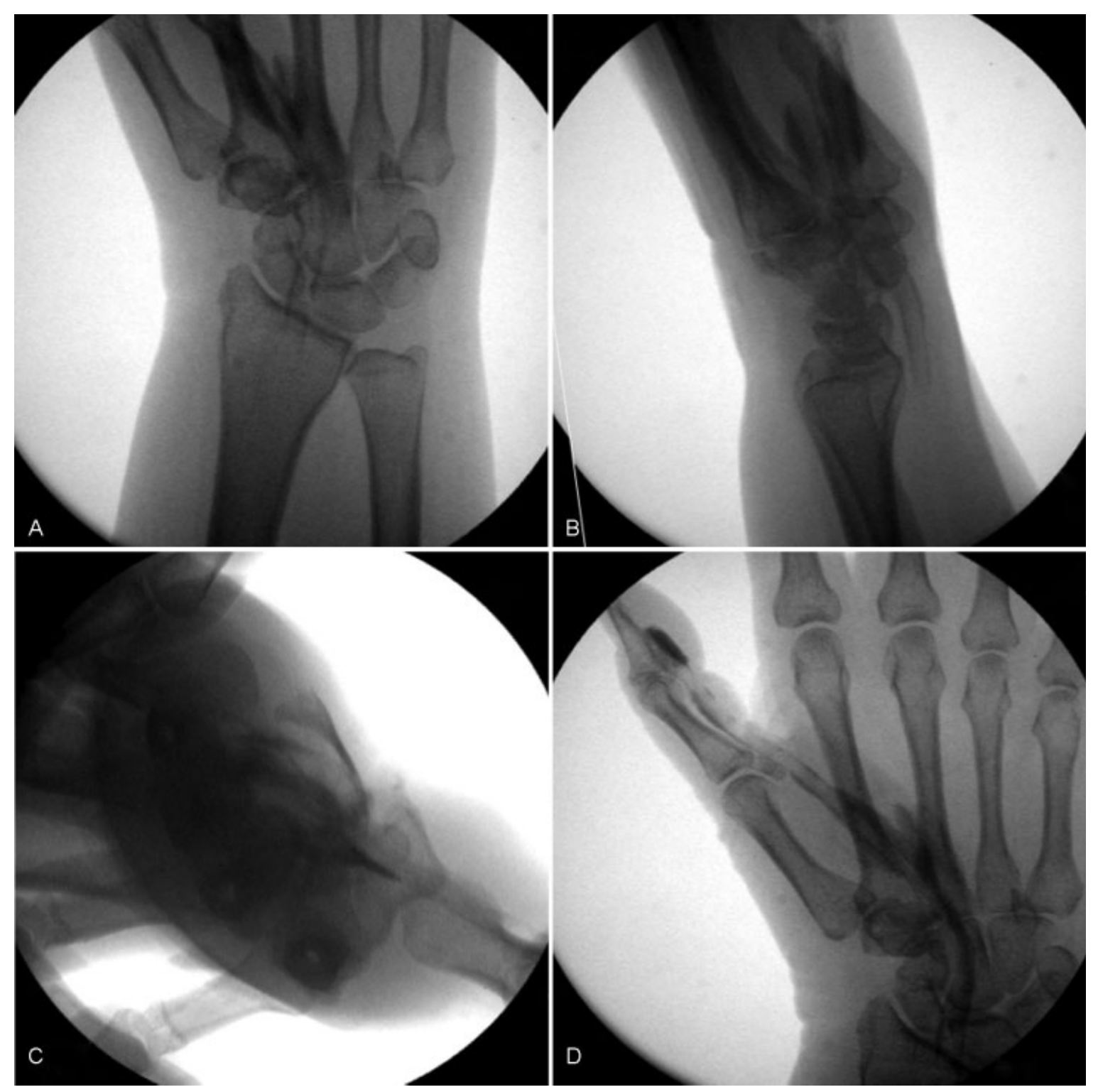

Fig. 4 Fluoroscopic (A) anteroposterior, (B) lateral, and (C) carpal tunnel images after a radial-sided injection of the carpal canal showing radioopaque dye located within the carpal canal. (D) Radio-opaque dye shown along the flexor pollicis longus flexor tendon sheath. 
The risk of iatrogenic injection into the underlying neurovascular structures resulting in exacerbation of symptoms, permanent neurologic deficit, or vascular injury is a known potential complication of this procedure. Various authors have performed studies and recommended techniques based on safety. Gelberman et $\mathrm{al}^{15}$ determined the MN to be $6-\mathrm{mm}$ wide and 2.1-mm thick at a location $1 \mathrm{~cm}$ proximal to the distal wrist flexion crease and advocated for injection through the FCR tendon, with further studies by Dubert and Racasan ${ }^{17}$ and Racasan and Dubert ${ }^{14}$ supporting this approach. Moving ulnarly, Graham et $\mathrm{al}^{4}$ suggested needle placement just ulnar to the FCR, and Koo and Szabo ${ }^{18}$ advised injection between the FCR and PL. Additional recommended techniques include needle placement ulnar to the PL, ${ }^{1,5,8,9,12,19}$ as well as between the PL and FCU. ${ }^{13,15,20}$

We argue, however, that due to differences in patient size, variations in location of neurovascular structures relative to superficial landmarks, and the wide range of superficial landmarks relative to total wrist width observed in the present study, a standardized technique based on total wrist width may result in a lower incidence of iatrogenic injury. Our results demonstrate that superficial landmarks vary widely among patients. For example, the PL was located ulnar to the $\mathrm{MN}$ in $53 \%$ of patients with a mean distance of $4.21 \mathrm{~mm}$ (range, $1-31 \mathrm{~mm}$ ), radial in $25 \%$ with a mean distance of $5.0 \mathrm{~mm}$ (range, $1-8 \mathrm{~mm}$ ), directly overlying the $\mathrm{MN}$ in $11 \%$, and not present in $11 \%$ of subjects. These findings indicate that a carpal tunnel injection placed just ulnar to the PL as described above could potentially result in inadvertent damage to the MN in $36 \%$ of patients who have a PL either directly overlying or radial to the MN. Our data also suggest that any technique that relies on the PL as a superficial landmark cannot be used in $11 \%$ of patients who are without this muscle.

Although such wide variation among superficial landmarks exists, our results regarding the locations of the underlying neurovascular structures as a function of total wrist width are consistent regardless of patient gender or wrist laterality. Analysis of the locations of the RA, MN, and UB between male and female specimens showed no statistically significant differences (RA: $p=0.91$; MN: $p=0.86$; UB: $p=0.24$ ) based on percentage of wrist width. Our findings are further generalizable in that there was minimal difference in wrist width between right and left specimens. Although right wrists were, on average, slightly larger than those on the left, this difference was not significant ( $p=0.783$ ). By measuring total wrist width and using a percentage of this value to determine the injection site, we have attempted to provide guidelines for carpal tunnel injections that can be easily reproduced regardless of variation in a patient's superficial anatomy.

Moreover, the reproducibility of injection delivery has been infrequently evaluated and results have varied. Minamikawa et $\mathrm{al}^{21}$ performed a series of carpal tunnel injections in 16 fresh cadaver forearms using methylene blue to compare the efficacy of fluid delivery while also minimizing risk of intraneural injection. The specimens were dissected and dye diffusion was quantified among four groups. They demonstrated the best diffusion was achieved when $2 \mathrm{~mL}$ of methylene dye was injected $3 \mathrm{~cm}$ proximal to the distal wrist flexion crease ulnar to the PL in line with the third web space. This technique was found to be superior to more radialsided injections located between the PL and FCR, or injections performed $1 \mathrm{~cm}$ proximal to the distal wrist flexion crease. In one radial-sided approach (6\%), the carpal tunnel was missed entirely. Wood ${ }^{12}$ demonstrated that an experienced injector missed the carpal tunnel $8 \%$ of the time (2/26 wrists). However, this study involved placing needles in the standard injection position and dissecting the wrist to find the tip position. No fluid was injected, and their study rationalized that the probable miss rate would be lower in clinical practice as the surgeon would detect resistance to injection and reposition the needle accordingly.

Our results suggest the carpal tunnel may be reliably injected from either the VR or VU approach. Radial-sided injections placed at either 30 or $33 \%$ of total wrist width resulted in no penetrations of the RA or MN. With the VR approach, one can successfully inject fluid into the carpal tunnel with a low risk of iatrogenic injury to the underlying neurovascular structures, but the injector may expect to encounter some soft tissue resistance to the injection, as well as the unanticipated effect of injecting fluid into the FPL tendon sheath. Inadvertent injection into the FPL tendon sheath occurred $80 \%$ of the time with a radial-sided approach. Alternatively, penetration of critical structures using VU injections resulted in two penetrations of the $\mathrm{MN}$ at $60 \%$ of total wrist width and six penetrations into the UB at $66 \%$. The VU injection showed equal efficacy in fluid delivery within the carpal canal, had less soft tissue resistance to the injection, and eliminated the likelihood of injecting into the FPL tendon sheath.

While this study resulted in novel findings and proposed a new standardized approach for carpal tunnel injections based on total wrist width, the experimental design had its limitations. The study was based on a cadaveric model, and as such, lacked clinical outcomes' data produced by similar retrospective or prospective trials. This pilot study, however, was necessary to provide preliminary anatomical data, which will be used to design a more rigorous prospective study. Another inherent limitation based on a cadaveric model is that intraneural injection cannot be easily detected in the absence of patient feedback. In the clinical setting, an intraneural injection into a median or ulnar nerve would likely produce pain and/or paresthesias, acting as an immediate indication of improper needle placement. The location and direction of the needle would then be appropriately adjusted, potentially altering outcomes. However, a patient may misinterpret these symptoms as simply being part of the injection itself, unable to differentiate pain from the injection versus the needle being placed intraneurally. Also, in certain situations (e.g., a sedated patient undergoing a carpal tunnel injection for a carpal tunnel release), this sensory feedback may not be present. Using a standardized approach would be helpful as a starting point for carpal tunnel injection regardless of patient size or variations in surface anatomy. 
Although carpal tunnel injections are a relatively safe and effective procedure, the ideal location to minimize risk of iatrogenic injury to the underlying RA, MN, and UB remains controversial in the literature. Our data demonstrate a wide variability in commonly described superficial landmarks, such as the FCR, PL, and FCU. This variability may be responsible for inconsistent placement of the injection within the carpal canal and an increased risk of adverse events. While further prospective studies are warranted to more rigorously evaluate the efficacy and clinical outcomes of these guidelines, our data demonstrate radial-sided injections placed at one-third of total wrist width, as measured from the radial styloid, offer a safe and reliable standardized approach to the carpal canal that eliminates the wide variability associated with superficial musculature. Our results also demonstrate an ulnar-sided injection using $60 \%$ of total wrist width is relatively safe, although moving further in an ulnar direction should be avoided to prevent injury to the UB.

\section{Note}

This article does not contain any studies with human participants or animals performed by any of the authors.

\section{Conflict of Interest}

None.

\section{Acknowledgment}

The authors received support from UL1 TR000445 from National Center for Advancing Translational Sciences (NCATS)/ National Institute of Health (NIH) for utilization of Research Electronic Data Capture (REDCap).

\section{References}

1 Edgell SE, McCabe SJ, Breidenbach WC, LaJoie AS, Abell TD. Predicting the outcome of carpal tunnel release. J Hand Surg Am 2003;28(2):255-261

2 Feuerstein M, Burrell LM, Miller VI, Lincoln A, Huang GD, Berger R. Clinical management of carpal tunnel syndrome: a 12-year review of outcomes. Am J Ind Med 1999;35(3):232-245

3 Gonzalez MH, Bylak J. Steroid injection and splinting in the treatment of carpal tunnel syndrome. Orthopedics 2001;24(5):479-481
4 Graham RG, Hudson DA, Solomons M, Singer M. A prospective study to assess the outcome of steroid injections and wrist splinting for the treatment of carpal tunnel syndrome. Plast Reconstr Surg 2004;113(2):550-556

5 Green DP. Diagnostic and therapeutic value of carpal tunnel injection. J Hand Surg Am 1984;9(6):850-854

6 Katz JN, Keller RB, Simmons BP, et al. Maine Carpal Tunnel Study: outcomes of operative and nonoperative therapy for carpal tunnel syndrome in a community-based cohort. J Hand Surg Am 1998; 23(4):697-710

7 McGrath MH. Local steroid therapy in the hand. J Hand Surg Am 1984;9(6):915-921

8 Weiss A-P, Sachar K, Gendreau M. Conservative management of carpal tunnel syndrome: a reexamination of steroid injection and splinting. J Hand Surg Am 1994;19(3):410-415

9 Phalen GS. The carpal-tunnel syndrome. Seventeen years' experience in diagnosis and treatment of six hundred fifty-four hands. J Bone Joint Surg Am 1966;48(2):211-228

10 Ozdoğan H, Yazici H. The efficacy of local steroid injections in idiopathic carpal tunnel syndrome: a double-blind study. $\mathrm{Br} \mathrm{J}$ Rheumatol 1984;23(4):272-275

11 Schuchmann JA, Melvin JL, Duran RJ, Coleman CR. Evaluation of local steroid injection for carpal tunnel syndrome. Arch Phys Med Rehabil 1971;52(6):253-255

12 Wood MR. Hydrocortisone injections for carpal tunnel syndrome. Hand 1980;12(1):62-64

13 Frederick HA, Carter PR, Littler JW. Injection injuries to the median and ulnar nerves at the wrist. J Hand Surg Am 1992;17(4): 645-647

14 Racasan O, Dubert T. The safest location for steroid injection in the treatment of carpal tunnel syndrome. J Hand Surg [Br] 2005;30(4): 412-414

15 Gelberman RH, Aronson D, Weisman MH. Carpal-tunnel syndrome. Results of a prospective trial of steroid injection and splinting. J Bone Joint Surg Am 1980;62(7):1181-1184

16 Grassi W, Farina A, Filippucci E, Cervini C. Intralesional therapy in carpal tunnel syndrome: a sonographic-guided approach. Clin Exp Rheumatol 2002;20(1):73-76

17 Dubert T, Racasan O. A reliable technique for avoiding the median nerve during carpal tunnel injections. Joint Bone Spine 2006; 73(1):77-79

18 Koo JT, Szabo RM. Compression neuropathies of the median nerve. J Am Soc Surg Hand 2004;4(3):156-175

19 McConnell JR, Bush DC. Intraneural steroid injection as a complication in the management of carpal tunnel syndrome. A report of three cases. Clin Orthop Relat Res 1990;(250): 181-184

20 Kay NRM, Marshall PD. A safe, reliable method of carpal tunnel injection. J Hand Surg Am 1992;17(6):1160-1161

21 Minamikawa Y, Peimer CA, Kambe K, Wheeler DR, Sherwin FS. Tenosynovial injection for carpal tunnel syndrome. J Am Soc Surg Hand 1992;17(1):178-181 